\title{
Protective effect of pneumococcal vaccine against death by pneumonia in elderly
} subjects

\author{
A. Vila-Córcoles*, O. Ochoa-Gondar*, C. Llor*, I. Hospital*, \\ T. Rodríguez ${ }^{\#}$ and A. Gómez*
}

ABSTRACT: The present study assessed the effectiveness of the 23-valent pneumococcal polysaccharide vaccine to prevent pneumonia and death in older adults in a first-time report between January and December 2002.

A prospective cohort study was conducted including all individuals $\geqslant 65$ yrs of age assigned to one of eight primary care centres in Tarragona, Spain $(n=11,241)$. The primary outcomes were community-acquired pneumonia (hospitalised or outpatient) and death from pneumonia. All pneumonias were validated by checking clinical records. The association between the pneumococcal vaccination and the risk of each outcome was evaluated by means of multivariate Cox proportional-hazard models, adjusted by age, sex, influenza vaccination status, comorbidity and immunological status.

Pneumococcal vaccination did not alter the risk of hospitalisation from pneumonia (hazard ratio (HR): 0.80 ; 95\% confidence interval $(\mathrm{Cl}): 0.50-1.28)$ or overall pneumonia (HR: 0.86 ; $95 \% \mathrm{Cl}: 0.56-$ 1.31), but the vaccine was associated with considerable reductions of death risk from pneumonia (HR: 0.28; 95\% Cl: 0.09-0.83).

In conclusion, these results suggest that pneumococcal polysaccharide vaccine may not be effective in reducing the incidence of pneumonia, but may be able to diminish the severity of the infection. These findings support the effectiveness of the pneumococcal polysaccharide vaccine to prevent mortality caused by pneumonia in older adults, providing a new argument to recommend systematic vaccination in the elderly.

KEYWORDS: Effectiveness, elderly, mortality, pneumococcal vaccine, pneumonia

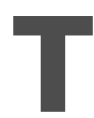
he 23-valent polysaccharide pneumococcal vaccine (PPV) has been available since 1983 and is currently recommended for use in the elderly and high-risk groups [1, 2]. However, despite numerous studies, its efficacy in the prevention of pneumococcal infections and other clinically relevant medical outcomes is not clear.

Several observational trials have shown a protective effect on preventing pneumococcal bacteraemia $[3,4]$, some have not shown this effect [5, 6], while others have reported little increased risk of being hospitalised or suffering from overall pneumonias $[7,8]$.

A meta-analysis limited to studies in more developed countries showed a protective effect to prevent pneumococcal bacteraemia, but a

For editorial comments see page 982 . significant protective effect against pneumonia amongst elderly subjects or high-risk groups was not found [9]. A recent Cochrane review [10] concluded that, while polysaccharide pneumococcal vaccines do not appear to reduce the incidence of pneumonia or death in adults (with or without chronic diseases) or in the elderly, the evidence from nonrandomised studies suggests that polysaccharide vaccines are effective in reducing the incidence of the more specific outcome (invasive pneumococcal disease) among adults and immunocompetent elderly subjects aged $\geqslant 55$ yrs.

The latest studies on the vaccine have not clarified the controversy regarding the effectiveness of this vaccine. In a large retrospective cohort study including $>47,000$ patients $>65$ yrs of age, JACKSON et al. [8] found that the effectiveness in preventing pneumococcal bacteraemia

\section{AFFILIATIONS}

*EVAN-65 Group of Primary Care Service Tarragona-Valls, Institut Catalá de la Salut, Tarragona, and \# Dept of Statistic and Research of Jordi Gol i Gurina, Barcelona, Spain.

\section{CORRESPONDENCE}

A. Vila-Corcoles

Institut Català de la Salut, Prat de la Riba 39

Tarragona 43001

Spain

Fax: 34977226411

E-mail: avila.tarte.ics@gencat.net

Received:

March 152005

Accepted after revision:

August 142005

SUPPORT STATEMENT

This study is supported by a grant from the Health Research Fund (FIS) of the Spanish Ministry of Health and Consumer Affairs.
European Respiratory Journal Print ISSN 0903-1936 Online ISSN 1399-3003 
was $56 \%$. They also observed an unexplained $14 \%$ increase in the risk of hospitalisation due to pneumonia among vaccinated subjects. The following two case-control studies have reported contradictory results. BENIN et al. [11] did not find any significant protective effect of PPV in pneumococcal invasive disease in Navajo adults (group with high incidence of pneumococcal infections). In contrast, DomINGUEZ et al. [12] found an effectiveness of $72 \%$ to prevent invasive disease amongst Spanish people aged $>65$ yrs.

Despite these inconclusive results, the use of 23-valent PPV is recommended in high-risk groups and the general elderly population on the basis that the effectiveness of the vaccine in preventing pneumococcal bacteraemia among these two groups has been demonstrated. There is sufficient evidence to justify widespread use $[1,2,13]$.

In the current authors' opinion, it is not clear whether or not the 23-valent PPV reduces the different risks associated with pneumococcal infections. Thus, long-term prospective studies based on clinical data are required to determine the real efficacy of PPV to prevent related events such as invasive disease, pneumonia and death in different population groups.

In Catalonia (Spain), the systematic recommendation for free pneumococcal vaccination in all elderly subjects $>65$ yrs of age (with or without risk factors) was introduced in 1999 [2]. This study was planned for 3 yrs and was started on January 1, 2002 [14]. The effectiveness of the 23-valent PPV in preventing pneumonia and death among people aged $>65$ yrs was assessed in a first-time report from January 1, to December 31, 2002

\section{METHODS}

A prospective cohort study of community-dwelling subjects aged $>65 \mathrm{yrs}$, assigned to one of eight primary care centres (PCC) in Tarragona, Catalonia $(n=11,241)$ was conducted. All cohort members were followed until: enrolment from the PCC ceased; death or any event occurred; or until the end of the follow-up.

In this early report, the primary end points resulting from the first 12 months of the survey (from January 1, to December 31, 2002) were analysed. The present study used the institution's general vaccinations database (from 1998) and the database of electronic patient records (which contained immunisation registries) of the eight participating PCCs to identify pneumococcal and influenza vaccinations.

The primary outcomes were community-acquired pneumonia (CAP; with hospitalisation or outpatient), death caused by pneumonia and death from any cause. Hospitalisations for pneumonia were identified on the database of hospital admissions from discharge codes according to the International Classification of Diseases 9th Revision (ICD-9: 480 to 487.0 ). All the cases were validated by checking the medical records. CAP was considered if, on review of the medical record review, the physician confirmed that this diagnosis was correct and was not a re-admission, nosocomial pneumonia or any another diagnosis. Outpatient pneumonia was defined as a primary care or emergency visit (not hospitalised) with an ICD-9 code registered for pneumonia in the PCC databases. In all the cases the medical records were reviewed to validate the diagnosis using the same criteria as for hospitalised pneumonia. In all cases, the presence of an infiltrate on chest radiographs was necessary to validate the diagnosis.

The presence or absence of any comorbidity (chronic heart disease, diabetes mellitus, chronic lung disease, high blood pressure, obesity, smoking) was determined by review of the ICD-9 codes reformed in the databases and clinical records of each PCC. Immunocompromise was a composite variable defined by the presence of any one of the following: cancer (solid organ or haematological neoplasia); chronic severe nephropathy (nephrotic syndrome, renal failure, dialysis or transplantation); chronic severe liver disease (cirrhosis); anatomical or functional asplenia; AIDS; and long-term corticosteroid therapy $\left(20 \mathrm{mg} \cdot \mathrm{day}^{-1}\right.$ of prednisone $)$ or another immunosuppressive medication $[8,12]$.

The incidence of each event was calculated as persons-year, considering that in the denominator the total persons-time for the study period was simply the sum of the persons-time contributed to each individual. Multivariable Cox proportional hazards models, with a time-varying covariable, were used to evaluate the association between having received the pneumococcal vaccine and the time to first outcome during the study period. Pneumococcal vaccination status was a timevarying covariable and persons were considered to be vaccinated 14 days after vaccine administration. Other covariables were defined on entry into the study.

The proportional hazard assumptions were assessed, adding the covariate by time interactions to the model and plotting the scaled and smoothed Schoenfeld residuals obtained from the main effects model where possible. The current authors also checked for confounding factors, interactions and multicollinearity among the independent variables.

Multivariable Cox models began with all variables significant in the univariate analysis at the 25 per cent level, as well as other variables that were judged of clinical importance.

\section{RESULTS}

The 11,241 cohort members were observed for a total of 11,025 persons-year, of which 5,005 (46.3\%) persons-year followed 23valent PPV. In total, 4,986 subjects were vaccinated before the study started, while $4,314(87 \%)$ had received 23 valent-PPV during the previous 2 yrs. Of the 6,255 subjects who had not received 23 valent-PPV before entering the study, $720(11 \%)$ were vaccinated during the 12 -month study period. These 720 subjects contributed to the analysis for a total of 708.5 personsyear (527.6 persons-year in the unvaccinated group and 180.9 persons-year in the vaccinated group). The baseline characteristics of cohort members are shown in table 1.

During the 12 months follow-up, CAP was observed in 117 subjects (22 outpatient and 95 hospitalisations). Among the cohort members there were 385 deaths from any cause (18 deaths caused by pneumonia). Therefore, there was an incidence (per 1,000 persons-year) of 10.62 for overall pneumonia (8.62 hospitalisations from pneumonia and 2.01 outpatient pneumonia), and a death rate from any cause of 34.92 per 1,000 . 


\begin{tabular}{|c|c|c|c|}
\hline Characteristics & $\begin{array}{l}\text { Unvaccinated } \\
\text { before entry }\end{array}$ & $\begin{array}{l}\text { Vaccinated } \\
\text { before entry }\end{array}$ & p-value ${ }^{\#}$ \\
\hline Subjects $n$ & 6255 & 4986 & \\
\hline \multicolumn{4}{|l|}{ Age group yr } \\
\hline $75-84$ & $1780(28.5)$ & $2080(41.7)$ & \\
\hline$\geqslant 85$ & $572(9.1)$ & $604(12.1)$ & \\
\hline \multicolumn{4}{|l|}{ Sex } \\
\hline Male & $2716(43.4)$ & $2176(43.6)$ & 0.814 \\
\hline Female & 3539 (56.6) & $2810(56.4)$ & \\
\hline Influenza vaccination previous yr & $1917(30.6)$ & $4115(82.5)$ & $<0.001$ \\
\hline Chronic lung disease & $598(9.6)$ & $701(14.1)$ & $<0.0001$ \\
\hline Smokers & $569(9.1)$ & $361(7.2)$ & $<0.0001$ \\
\hline Immunocompromised status & $652(10.4)$ & $794(15.9)$ & $<0.0001$ \\
\hline
\end{tabular}

Data are presented as $n(\%)$, unless otherwise stated. ${ }^{\#}:$ p-values were calculated with the Chi-squared test; $"$ : the median ages of the unvaccinated and vaccinated subjects were 73.4 and 75.9 , respectively.

An aetiological study was carried out in 89 (76.1\%) of the 117 pneumonias. Streptococcus pneumoniae was identified in 12 cases (blood culture $n=9$, sputum culture $n=3$ ) and other aetiological agents were identified in 25 cases. During the influenza season (January 1, to May 15) 50 cases of pneumonia were observed (12.1 cases per 1,000 inhabitants-year), and 67 pneumonias were observed during the rest of the year $(9.7$ per 1000).

The initial bivariate analysis showed higher crude rates for hospitalisation and overall pneumonia among vaccinated subjects, but it also showed lower crude rates for death among these vaccinated subjects.

The mortality caused by pneumonia was $15.4 \%$ of overall pneumonias $(20.3 \%$ among unvaccinated and $10.4 \%$ among vaccinated; $\mathrm{p}=0.134)$. Of the 18 deaths from pneumonia, seven occurred during the influenza season (two among those vaccinated with 23-valent PPV and influenza vaccine, two among those vaccinated against influenza, but not vaccinated with PPV, and three among those not vaccinated with either).

In total, six cases of pneumococcal bacteraemia were observed among unvaccinated subjects and three cases among vaccinated subjects (hazard ratio (HR): $0.63 ; 95 \%$ confidence interval (CI): 0.12-2.79).

In the multivariable analysis, pneumococcal vaccination did not significantly alter the risk of CAP. The pneumococcal vaccination showed a little, nonsignificant reduction in the risk for hospitalisation (HR: 0.81; 95\% CI: 0.51-1.30) and overall pneumonia (HR: 0.85; 95\% CI: 0.56-1.31). However, the reception of the 23-valent PPV was associated with a significant reduction of death from pneumonia (HR: 0.28; 95\% CI: 0.09 0.80 ) and death from any cause (HR: $0.67 ; 95 \%$ CI: $0.54-0.83$ ).
The values of different results for each event are shown in table 2. Multicollinearity in predictor variables was not found. The proportionality of hazards was observed in all the predictor variables in each model. Influenza vaccine was found to be a confounder variable in hospitalisation and overall pneumonia. All the predictors appeared statistically significant at the level of $5 \%$, except the confounder.

\section{DISCUSSION}

The present authors undertook a long prospective study to evaluate the controversial effectiveness of the 23-valent PPV in older adults. Although it is not randomised, the large size of the cohorts, together with the adjustment of the main confounding factors, in the multivariate analysis form an adequate basis to assess the potential health effects of the pneumococcal vaccination in the elderly population.

The vaccinated group and the nonvaccinated group differed significantly in all baseline characteristics except sex in the current study. The vaccinated subjects were older and had more comorbidity than the nonvaccinated subjects. In spite of the current authors adjusting the analysis for these factors, it is possible that the effect of the vaccine could have been underestimated for this reason. The vaccinated group had larger influenza vaccination rates than the nonvaccinated group, and this was a confounding factor. The adjustment for the influenza vaccine status was also included in the Cox models. The reception of this vaccine has also demonstrated effectiveness to reduce hospitalisations for pneumonia and death [15], and this must be taken into account in the analysis.

In this first-time study the present authors have not been able to document a significant effect of PPV on preventing 
TABLE 2 Incidence and risk of hospitalisation for pneumonia, overall pneumonia, death from pneumonia and death from any cause in relation to pneumococcal vaccination status\# ${ }^{\#}$

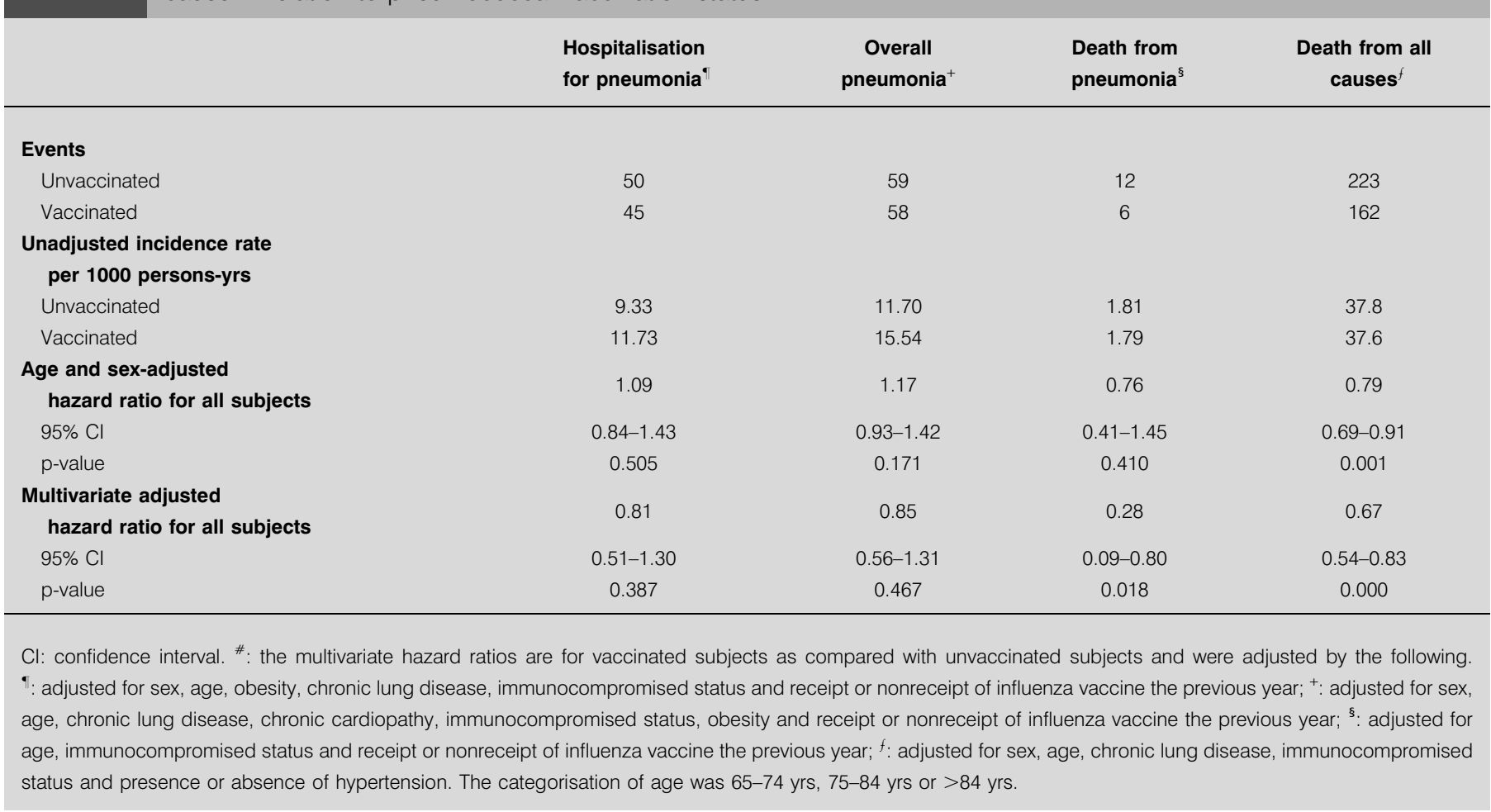

hospitalisation or overall pneumonia. However, the main contribution of the work is that a significant protective effect of the 23-valent PPV against death caused by pneumonia, among elderly adults living in the community, has been found.

A little, but nonsignificant, effect of the 23-valent PPV to reduce hospitalisation from pneumonia (19\%) or overall pneumonia $(15 \%)$ was found.

Although a significant decrease in the risk for overall pneumonia was not detected, the possibility of the vaccine having some effect against pneumococcal pneumonia (with or without bacteraemia) cannot be excluded. The incidence of pneumococcal pneumonia was low. For this reason, although 23-valent PPV seems to reduce the risk of pneumococcal bacteraemia (vaccinated $n=3$, unvaccinated $n=6$ ), the current study had very limited power to detect this possible protective effect.

The association between vaccination and pneumococcal pneumonia without bacteraemia could not be directly evaluated because an aetiological agent was not identified in most cases of CAP [16]. However, assuming that S. pneumoniae is the most common cause of CAP [16], the present results show that 23-valent PPV did not have a great protective effect against nonbacteraemic pneumococcal pneumonia.

The effectiveness of PPV in the elderly and high-risk groups is controversial. The recommendation of 23-valent PPV is based on several clinical trials (i.e. trials before 1980) and some observational studies, which found a protective effect against bacteraemia and invasive disease [10]. Several meta-analyses have evaluated this problem and, despite inconclusive results, it has generally been observed that PPV is effective in preventing bacteraemia and possibly pneumococcal pneumonia in high-risk subjects $<55$ yrs of age. However, according to some meta-analysis, the effectiveness of 23-valent PPV has not been demonstrated in elderly subjects or high-risk individuals $[9,17-20]$.

A Cochrane review [10] valued a vaccine effectiveness of $53 \%$ $(41-63 \%)$ to prevent bacteraemia or invasive pneumococcal disease, but this review has also concluded that the vaccine did not prove a significative effect in preventing either pneumonia $(-15-20 \%)$ or death $(-9-12 \%)$. In the last published metaanalysis, MELEGARO and EDMUNDS [20] concluded that the PPV provides $65 \%$ protection against invasive pneumococcal disease in the general elderly population, having a moderate $20 \%$ effect in the high-risk elderly. They also concluded that the vaccine could have little or null effect against pneumonia in the general elderly population (-20-16\%).

Several studies have shown a reduction in risk of death by pneumonia among younger adults and institutionalised elderly subjects vaccinated with PPV [21, 22], but an effect on mortality from pneumonia has not been demonstrated for high-risk patients or noninstitutionalised older adults to date $[9,17-20]$. In 1998, in a randomised clinical trial amongst 690 individuals aged $>50$ yrs with high-risk (previous pneumonia), ORTQVIST et al. [23] observed a nonsignificant reduction of $31 \%$ in the mortality caused by pneumococcal diseases among subjects vaccinated with 23-valent PPV. In a retrospective cohort study published in 1999, NicHOL et al. [24] observed that the pneumococcal vaccination produced a $47 \%$ reduction in hospitalisations and 29\% (9-44\%) deaths among 1,898 patients with chronic obstructive pulmonary disease. Recently, in a 
nonrandomised large scale intervention including $>250,000$ individuals aged $>65$ yrs in Stockholm (Sweden), CHRISTENSON et al. [25] observed an additive effect of influenza and pneumococcal vaccinations in reducing mortality by $35 \%$ (22-44\%) caused by pneumonia, but this effect was not significant in patients who had only received the 23-valent PPV.

The present findings are compatible with the data reported by these studies and previous meta-analysis. They could provide a valid explanation to the reason why contradictory results regarding this vaccine have been found in previous studies. The majority of meta-analyses did not find a significant protective effect of PPV against noninvasive disease or overall pneumonia in the elderly. It is possible that PPV could not avoid the infection due to Pneumococcus in many elderly subjects. However, PPV may decrease the severity of pneumococcal infection by preventing invasion of the bloodstream and the incidence of the bacteraemia (as reported in several observational studies) and it may decrease mortality (as found the current study).

The reduction of overall deaths was significant. It is possible that some deaths could be caused by another pneumococcal infection not considered in the current study (e.g. meningitis) or by complications after pneumonias. However, a possible selection bias nonadjusted in the multivariable analysis cannot be rejected.

In conclusion, the present authors emphasise that this study should be considered as an interim analysis, since questions remain unanswered. It is important to estimate accurately the level of protection in high- and low-risk individuals, because these two groups are at the centre of the current debate on whether or not to extend the vaccination programme to all elderly populations $[13,20]$.

\section{ACKNOWLEDGEMENTS}

The authors would like to thank to J. Fort, M. Piqueras, J. Grifoll, A. Garcia, A. Ansa, P. Mollonedo, J.L. Pinyol and all family physicians of Primary Care Centers of Tarragona-Valls (Spain) for their inestimable help in the production of this paper.

\section{REFERENCES}

1 Centers for Disease Control and Prevention. Prevention of pneumococcal disease. Recommendations of the advisory committee on immunisation practices (ACIP). MMWR 1997; 46: 1-24.

2 Salleras Ll, Urbiztondo Ll, Fernández $\mathrm{N}$, et al. Pneumococcal vaccination in elderly people. Med Clin (Barc) 2001; 116: Suppl. 1, 18-23.

3 Shapiro ED, Berg AT, Austrian R, et al. The protective efficacy of polyvalent pneumococcal polysaccharide vaccine. N Engl J Med 1991; 325: 1453-1460.

4 Butler JC, Breiman RF, Campbell JF, et al. Pneumococcal polysaccharide vaccine efficacy. An evaluation of current recommendations. JAMA 1993; 270: 1826-1831.

5 Simberkoff MS, Cross AP, Al IM, et al. Efficacy of pneumococcal vaccine in high-risk patients. Results of a
Veterans Administration Cooperative Study. N Engl J Med 1986; 315: 1318-1327.

6 Forrester HL, Jahnigen DW, LaForce FM. Inefficacy of pneumococcal vaccine in a high-risk population. Am J Med 1987; 83: 425-430.

7 Ortqvist A, Hedlund J, Burman LA, et al. Randomised trial of 23-valent pneumococcal capsular polysaccharide vaccine in prevention of pneumonia in middle-aged and elderly people. Swedish Pneumococcal Vaccination Study Group. Lancet 1998; 351: 399-403.

8 Jackson LA, Neuzil KM, Yu O, et al. Effectiveness of pneumococcal polysaccharide vaccine in older adults. $N$ Engl J Med 2003; 348: 1747-1755.

9 Mangtani P, Cutts F, Hall AJ. Efficacy of polysaccharide pneumococcal vaccine in adults in more developed countries: the state of the evidence. Lancet Infect Dis 2003; 3: 71-78.

10 Dear K, Holden J, Andrews R, Tatham D. Vaccines for preventing pneumococcal infection in adults. Cochrane Database Syst Rev 2003; 4: CD000422.

11 Benin AL, O'Brien KL, Watt JP, et al. Effectiveness of the 23valent polysaccharide vaccine against invasive pneumococcal disease in Navajo adults. J Infect Dis 2003; 188: 81-89.

12 Dominguez A, Salleras L, Fedson DS, et al. Effectiveness of pneumococcal vaccination for elderly people in Catalonia, Spain: a case-control study. Clin Infect Dis 2005; 40: 1250-1257.

13 Fedson DS, Liss C. Precise answers to the wrong question: prospective clinical trials and the meta-analyses of pneumococcal vaccine in elderly and high-risk adults. Vaccine 2004; 22: 927-946.

14 Vila Córcoles A, Ochoa Gondar O, Hospital Guardiola I, et al. EVAN-65 project: evaluation of the effectiveness of pneumococcus vaccination in the elderly population over 65. Aten Primaria 2004; 33: 149-153.

$15 \mathrm{Vu} \mathrm{T}$, Farish S, Jenkins M, Kelly H. A meta-analysis of effectiveness of influenza vaccine in persons aged 65 years and over living in the community. Vaccine 2002; 20: 1831-1836.

16 Almirall J, Bolibar I, Vidal J, et al. Epidemiology of community-acquired pneumonia in adults: a populationbased study. Eur Respir J 2000; 15: 757-763.

17 Cornu C, Yzebe D, Leophonte P, Gaillat J, Boissel JP, Cucherat M. Efficacy of pneumococcal polysaccharide vaccine in immunocompetent adults: a meta-analysis of randomized trials. Vaccine 2001; 19: 4780-4790.

18 Fine MJ, Smith MA, Carson CA, et al. Efficacy of pneumococcal vaccination in adults. A meta-analysis of randomized controlled trials. Arch Intern Med 1994; 154: 2666-2677.

19 Moore RA, Wiffen PJ, Lipsky BA. Are the pneumococcal polysaccharide vaccines effective? Meta-analysis of the prospective trials. BMC Family Practice 2000; 1: 1.

20 Melegaro A, Edmunds WJ. The 23-valent pneumococcal polysaccharide vaccine. Part I. Efficacy of PPV in the elderly: a comparison of meta-analyses. Eur J Epidemiol 2004; 19: 353-363.

21 Riley ID, Lehmann D, Alpers MP, Marshall TF, Gratten H, Smith D. Pneumococcal vaccine prevents death from acute lower-respiratory-tract infections in Papua New Guinean children. Lancet 1986; 2: 877-881. 
22 Wagner C, Popp W, Posch M, et al. Impact of pneumococcal vaccination on morbidity and mortality of geriatric patients: a case-controlled study. Gerontology 2003; 49: 246-250.

23 Ortqvist A, Hedlund J, Burman LA, et al. Randomised trial of 23-valent pneumococcal capsular polysaccharide vaccine in prevention of pneumonia in middle-aged and elderly people. Lancet 1998; 351: 399-403.
24 Nichol HL, Baken L, Wuorenma J, Nelson A. The health and economic benefits associated with pneumococcal vaccination of elderly persons with chronic lung disease. Arch Intern Med 1999; 159: 2437-2442.

25 Christenson B, Hedlund J, Lundbergh P, Ortqvist A. Additive preventive effect of influenza and pneumococcal vaccines in elderly persons. Eur Respir J 2004; 23: 363-368. 